\title{
LA SALUD REPRODUCTIVA EN LA JUVENTUD CUBANA
}

\author{
NATIVIDAD GUERRERO BORREGO*
}

\begin{abstract}
RESUMEN
Una de las decisiones más trascendentales en la vida de las personas es la de tener o no hijos. La tendencia de las parejas cubanas, es de formar una familia en algún momento de sus vidas. Cuba alcanza los niveles más bajos de fecundidad dentro del escenario latinoamericano, adelantándose dos décadas al resto de la región.

El Centro de Estudios Sobre la Juventud cuenta con una basta experiencia en relación con la salud sexual y reproductiva de los jóvenes. El presente trabajo pretende aportar reflexiones que tributen al debate de la salud sexual y reproductiva en la población joven cubana.
\end{abstract}

PALABRAS CLAVE: SALUD, REPRODUCTIVA, JUVENTUD

* Doctora en Ciencias Psicológicas. Master en Sexología. Investigadora Titular y Profesora Titular de la Universidad de la Habana. Directora del Centro de Estudios Sobre la Juventud. E-Mail: ces@jovenclub.cu. 


\title{
A SAÚDE REPRODUTIVA DA JUVENTUDE CUBANA
}

\begin{abstract}
RESUMO
Ter ou não ter filhos é uma das decisões mais importantes na vida das pessoas. A tendência dos casais cubanos é de formar uma família em algum momento de suas vidas. Cuba possui os níveis mais baixos de fecundidade no cenário latino-americano, adiantando-se duas décadas ao restante da região. O Centro de Estudos Sobre a Juventude conta com vasta experiência em relação à saúde sexual e reprodutiva dos jovens. $O$ presente trabalho pretende trazer reflexões que contribuam com o debate sobre a saúde sexual e reprodutiva da população jovem cubana.
\end{abstract}

PALAVRAS-CHAVE: SAÚDE REPRODUTIVA, JUVENTUDE

\section{REPRODUCTIVE HEALTH IN THE YOUNG CUBAN}

\begin{abstract}
One of the most important decisions in the lives of people is to have children or not. The trend of Cuban couples is starting a family at some point in their lives. Cuba achieved the lowest levels of fertility in the Latin American scene, two decades ahead the rest of the region.

The Center for Youth Studies has vast experience in dealing with sexual and reproductive health of young people. This report aims to contribute reflections taxing debate on sexual and reproductive health in the young $\mathrm{Cu}-$ ban.
\end{abstract}




\section{INTRODUCCIÓN}

LA TOMA DE DECISIONES resulta un ejercicio cotidiano, pero no siempre consciente. Desde pequeños los seres humanos se comportan de una $\mathrm{u}$ otra forma, lo cual esta asociado esencialmente a la libertad, a la información y las condiciones con que cuenten para hacerlo.

Una de las decisiones más trascendentales en la vida de las personas es la de tener hijos o no tenerlos. La tendencia de las parejas, al menos en Cuba, es de formar una familia en algún momento de sus vidas, o la de tener un hijo/a aunque no se tenga una pareja estable. Este tema resulta polémico debido a las múltiples aristas asociadas a él, es un asunto universal que se matiza de acuerdo a la región o país del cual se trate.

En la primera década de la Revolución Cubana se produjo un incremento de la natalidad reconocida como «Baby Boom», lo que se consideró un comportamiento típico de contextos donde ocurren transformaciones sociales esperanzadoras. A finales de los años 70 comenzó a descender la fecundidad e incluso, los niveles de reemplazo poblacional, según información de la Oficina Nacional de Estadística (ONE), del Centro de Estudios Demográficos (CEDEM), y del Centro de Estudios Sobre la Mujer (CEM) (ONE, 2007).

Por esos tiempos dicha información no constituyó una alarma, pero a la altura del siglo XXI el análisis genera la necesidad de revertir la situación por la importancia que para el desarrollo de la población, la existencia y estabilidad del país tiene este complejo asunto, con el cual están responsabilizados una amplia gama de ministerios e instituciones.

Expertos cubanos señalan (ONE, CEDEM, CEM, 2007), que al disminuir los nacimientos se reduce la posibilidad de reemplazo; las niñas que nacen no son suficientes para mantener el desarrollo poblacional.

Desde la segunda mitad del siglo XX la fecundidad cubana había mostrado una cúspide temprana, en la que las menores de 20 años tuvieron una alta participación. Después de 1990 la estructura de la fecundidad tuvo una ligera dilatación que se mantiene hasta el presente siglo; aún continúa siendo el grupo de 20 a 24 años el que tiene mayores tasas, seguido del de 25 a 29 años. Se observa un incremento en el grupo de 30 a 34 años y las siguientes edades, incluyendo de los 40 a 44 años.

En relación con la estructura de la fecundidad, en nuestra isla podría distinguirse la creciente homogeneidad territorial que ha respondido a la amplia cobertura nacional en la implementación de políticas sociales encaminadas a elevar la calidad de vida de la población en asuntos de planificación familiar. Las provincias orientales, acompañadas de Pinar 
del Río (provincia más occidental del país) y el municipio especial Isla de la Juventud, hacen la mayor contribución en cuanto a los nacimientos, ello puede estar asociado al menor desarrollo socioeconómico en general de estos territorios.

Cuba alcanza los niveles más bajos de fecundidad dentro del escenario latinoamericano, adelantándose dos décadas al resto de la región. Esta situación se relaciona con los programas de desarrollo social instrumentados en el país, como los dirigidos a elevar la participación de la mujer en el desarrollo social. Estas políticas han favorecido el incremento del nivel educacional de las féminas, su nivel técnico-profesional y su preparación para planificar su descendencia.

El último decenio del siglo XX agudizó este proceso de descenso debido a la realidad socioeconómica que enfrentó el país, conocido como «Período Especial», incidiendo en la subjetividad de las parejas en cuanto a la formación de familias pequeñas y a la posposición de este proyecto de vida. Las estadísticas indican que las mujeres y parejas jóvenes en general, están posponiendo el momento del primogénito y disminuyendo la cantidad de hijos a tener.

En contraste con ello, se incrementa la esperanza de vida, los adultos mayores viven más años y se va invirtiendo la pirámide poblacional, según se constata en el último censo realizado en el 2002 (ver gráfico 1).

GRÁfICO 1: CUBA. PIRÁMIDE DE POBLACIÓN, POR SEXO Y GRUPOS DE EDADES

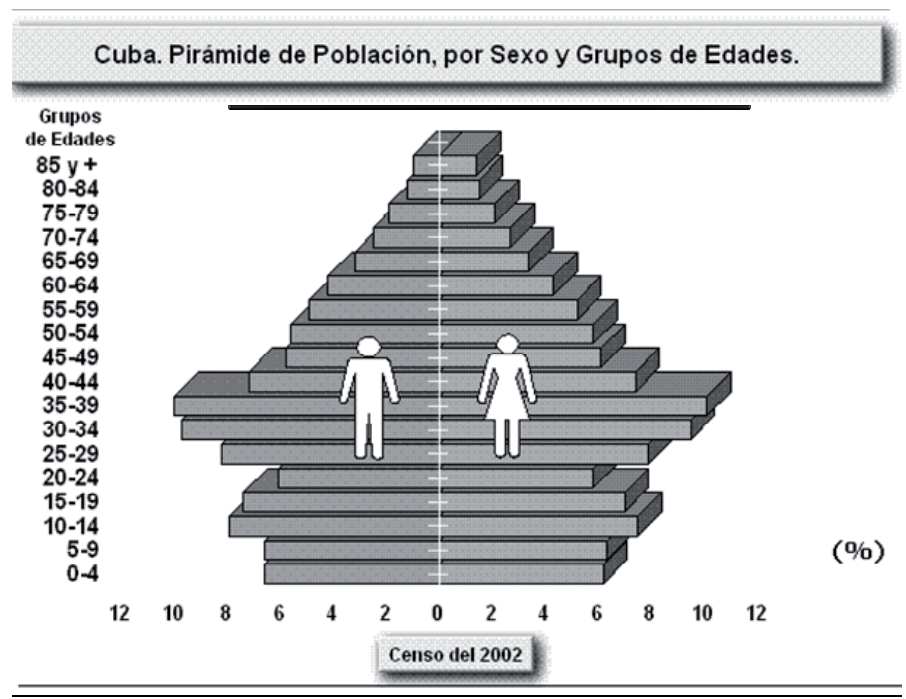

Fuente: MINSAP, 2009. 
Este proceso requiere una mirada holística y la revisión de algunas políticas sociales, de manera que se estimule la natalidad y se fortalezcan los servicios y la educación en torno al adulto mayor, sus particularidades y cuidados, además del énfasis en el valor de la riqueza de saberes y experiencias acumuladas en este sector poblacional en condiciones de seguir aportando a la sociedad. Hoy Cuba cuenta con una esperanza de vida de 77,3 (Sosa, 2009).

El Centro de Estudios Sobre la Juventud (CESJ) cuenta con una vasta experiencia en relación con la salud sexual y reproductiva de los jóvenes. Sin embargo, no cuenta con resultados que indaguen sobre aspectos de la Salud Reproductiva (SR) asociados a la baja fecundidad.

El presente trabajo pretende aportar reflexiones que tributen al análisis de la Salud Sexual y Reproductiva (SSR) en la población joven cubana, buscando ofrecer puntos de vista ineludibles en el debate puntual acerca de la fecundidad.

La salud reproductiva se define como un estado general de bienestar físico, mental y social asociados al sistema reproductivo, sus funciones y procesos, en el que intervienen hombre y mujer, según precisó la Organización Mundial de la Salud (OMS) en la Conferencia Internacional sobre Población y Desarrollo (CIPD,1994) celebrado en El Cairo. Entraña la capacidad de procrear y de decidir cuándo y cuántos hijos tener en caso de disponer tenerlos.

La salud reproductiva reconoce como un derecho humano que legitima la reproducción sin discriminación alguna, coerción ni violencia. Es identificada como derecho y defendida por feministas en Holanda a finales de la década del 70, las cuales reclamaron el derecho a decidir si la persona quería tener o no tener hijos. En este sentido se precisa que una vez tomada la decisión, no es lo mismo tener uno, que dos, o más. Cada hijo requiere un análisis en sí mismo, el primer hijo modifica la categoría de la persona deviniendo madre o padre, los otros hijos enriquecen esta condición y la matizan (Figueroa, 1996).

Hombres y mujeres tienen la posibilidad de tomar decisiones acerca de su reproducción libre y responsablemente, en cuanto al número de hijos, así como el espaciamiento entre ellos, pero este derecho no es reconocido universalmente. Esta afirmación tiene que ver con las diferentes políticas de salud pública, los niveles de ingreso y las prácticas sociales y culturales en las diversas regiones geográficas.

Existen en el mundo muchos obstáculos que limitan este proceso natural y que están relacionados con normas jurídicas, situaciones económicas e idiosincrasias. «Por lo menos 350 millones de personas que 
desean evitar el embarazo carecen de acceso a métodos de planificación familiar; 120 millones de mujeres carecen de servicios de salud reproductiva accesibles y aceptables y alrededor de medio millón de mujeres mueren cada año por causas relacionadas con el embarazo, donde el aborto es una de las causas más frecuentes» (Gran, 2005:12).

Lo anteriormente expresado está asociado a las determinantes sociales que tienen que ver con la salud reproductiva, pues en muchos países del mundo hay mujeres que son analfabetas o tienen muy baja escolaridad e información sobre el tema de la planificación familiar. Los bajos ingresos también inciden en el acceso a los métodos anticonceptivos y en la posibilidad de interrumpir un embarazo con garantía para la salud de la mujer.

La salud reproductiva forma parte de un complejo entramado social, que favorece o dificulta la descendencia humana. En este sentido vale hacer referencia a la construcción subjetiva de lo que implica ser madre y padre, a la negociación entre hombre y mujer en torno a la toma de la decisión de procrear o no y lo que ello genera desde la perspectiva de género, a los servicios de planificación familiar y a la dinámica familiar como red de apoyo, entre otras.

\section{UN ENFOQUE DE GÉNERO DESDE LA SALUD REPRODUCTIVA}

Una mirada desde el enfoque de género pone en desventaja a la mujer, pues en la negociación de la pareja, ella es quien tiene la responsabilidad de dar vida a la criatura concebida. Es ella la que asume la gestación por naturaleza y necesita de cuidados especiales para llegar a feliz término su embarazo.

La condición exclusiva de las mujeres de gestar la hace diferente del hombre; en muchas regiones del mundo se les ha negado la posibilidad de disfrutar su sexualidad aduciendo que la única función que le está asignada es la reproductiva. Ante este protagonismo en la reproducción-gestación, el hecho de no poder procrear en muchas regiones del planeta es altamente recriminado, devaluando y desestimando a la mujer que no tenga esta capacidad.

Los procesos de gestación y lactancia son los únicos momentos donde la pareja no asume igual responsabilidad; el hombre acompaña a su pareja durante la gestación, donde él tuvo la responsabilidad de determinar el sexo. Al concluir el embarazo y pasar a la maternidad-paternidad, vuelve la mujer a asumir, generalmente, el protagonismo ante la alimentación del bebé durante los primeros seis meses, si la lactancia es exclusi- 
va. Esto no debería significar que el padre se sienta relegado o se acomode a la idea de que es la mujer la máxima encargada del pequeño.

La legalización de los derechos sexuales de la mujer con plenitud para asumir todas las funciones de la sexualidad; esto es la función reproductiva, erótica y comunicativa aún son defendidas en algunas regiones de universo, todas las mujeres del planeta no gozan de libertad sexual. Este no es el caso de Cuba, en la isla hace 50 años se estimula la equidad entre los sexos y el derecho al placer, a la comunicación y a la reproducción protegidos desde entonces.

A la altura del siglo XXI se desestima el debate sobre este tema por considerarlo obsoleto, lo que no significa que en algún lugar del país con más bajo desarrollo socioeconómico y cultural, haya personas que conserven este tipo de prejuicios en el que se sigue discriminando a la mujer.

La sociedad cubana enfrenta desafíos en torno a esta problemática, pues si bien no constituye un problema social el considerar a la mujer sólo por su capacidad reproductiva, existen otros prejuicios que la limitan y desestiman; algunos tienen que ver con su participación más o menos activa en la relación sexual con su pareja, todavía es criticada cuando toma la iniciativa en cuanto a la satisfacción de sus deseos sexuales, es cuestionada mucho más fuerte que el hombre cuando no prioriza a sus hijos y jerarquiza algún asunto de otra índole.

Estas cuestiones han quedado socialmente rezagadas, están poco expuestas al debate público por ser más personales, por considerarlas erróneamente, un asunto íntimo que debe tratar cada pareja, cada familia, sin alcanzar a percibir que se expresa tanto a nivel individual, como familiar y social.

La salud reproductiva transita por diferentes criterios de legitimidad; está atravesada por los diversos credos que se practican en las distintas regiones que componen el universo, la condición genérica también lo matiza, pues se trata de un hecho en el que participan dos personas: hombre y mujer, y sólo una queda embarazada, es una acción relacional (Figueroa, 1996).

En esta misma dirección resultan varios análisis; pues una de las partes, la mujer, puede decidir tener descendencia sin contar con un varón, utilizando alguna tecnología como complemento. Implica una relación heterosexual y dentro de ella vale tener en cuenta si en la pareja ambos miembros tienen potencialmente posibilidades de reproducir, si ambos tienen interés en tener hijos y disposición para la negociación de tenerlos. 
Desde otra perspectiva, en cuestiones de salud reproductiva se identifica a la fecundidad como la variable sociodemográfica que la signa. Deviene expresión de la capacidad de reproducción humana, la cual está asociada a la anticoncepción y a la interrupción del embarazo, procedimientos que tienen efecto a través de los servicios gratuitos de Planificación Familiar existentes en el país.

La anticoncepción es el proceso mediante el cual se evita la fecundidad a través de métodos cada vez más inocuos para la salud. Algunas características de su desarrollo en el país evidencian que el $20 \%$ de las interrupciones que se producen ocurren por fallos anticonceptivos. Los dispositivos intrauterinos (DIU) tienen un margen de error de un $30 \%$ y las píldoras dependen de la responsabilidad de sus usuarias, que generalmente son jóvenes. Actualmente se avanza en la introducción de la píldora abortiva (Misoprostrol) como método menos agresivo para el organismo femenino (Sosa, 2008).

El país cuenta con una elevada cobertura anticonceptiva, lo cual indica la ocupación de sus autoridades gubernamentales en cuanto a este sensible tema. Existe un vasto empleo de los dispositivos intrauterinos, mientras el consumo del condón avanza sobre todo en el segmento juvenil. De acuerdo a la importancia que en estos tiempos se le concede, no llega a satisfacer las expectativas como barrera para evitar las ITS/VIH SIDA, además de los embarazos no deseados (ver gráfico 2).

La cobertura anticonceptiva responsabiliza nuevamente a la mujer, pues los métodos existentes son para ser utilizados esencialmente por las féminas, ellas reciben el impacto de estos métodos por inocuos que sean. Respecto a la esterilización masculina, son pocos los hombres que se someten a la vasectomía. En relación con el condón, suele ocurrir que las muchachas son las que, por lo general, lo llevan en sus bolsos.

La planificación familiar como servicio de la salud sexual y reproductiva, resulta esencial para el control de la fecundidad la cual tiene su expresión en los nacimientos, en el uso de métodos anticonceptivos y en las interrupciones de embarazos (abortos inducidos, espontáneos y regulaciones menstruales).

Las interrupciones de embarazos en Cuba, se concentran generalmente en jóvenes entre 18 y 19 años. El servicio que se brinda en el país garantiza la salud de las muchachas a través de la educación sexual, pero no se ha logrado elevar suficientemente la percepción de riesgo de estos procesos para el organismo. Esta afirmación hace vulnerable la fecundidad y la natalidad, pues aunque van disminuyendo las cifras de aborto en cualquiera de sus modalidades técnicas, es reiterativa la conducta de algunas 
jóvenes que no dan crédito a las posibles consecuencias de dichas interrupciones, sin que alcancen a asumirlas como el último recurso.

\section{GRÁFICO 2: PORCENTAJE DE COBERTURA ANTICONCEPCIONAL SEGÚN MÉTODOS. CUBA 2008}

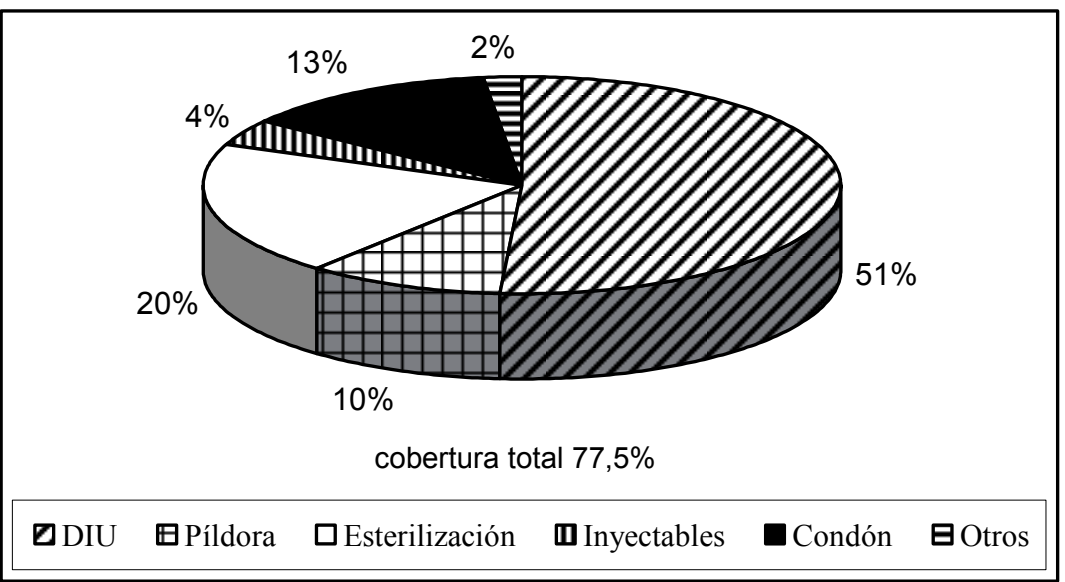

Fuente: MINSAP, 2009.

Cuba presta atención a las cifras relacionadas con esta problemática, pues está demostrada la garantía de la salud de las mujeres que se someten a estos procedimientos. Algunas muchachas no prestan atención a las posibles consecuencias que pueden tener estas operaciones y dejan al Estado la responsabilidad de «lo que pueda pasar». Los adultos por su parte, educan insuficientemente a sus menores, y en estos temas suelen dejar «a otros» esta responsabilidad.

Entre las consecuencias de las interrupciones de un embarazo se encuentran los efectos silentes que aparecen a largo plazo. Este suceso puede provocar inflamación pélvica, infecciones, hemorragias, lesiones traumáticas, etc. Se sabe que el $60 \%$ de las mujeres infértiles, se han sometido alguna vez a un aborto; lo cual sólo es un alerta que no niega alguna relación aún no corroborada como causa-efecto (Sosa, 2007).

El aborto deviene polémica internacional en tanto debate político, social, religioso, moral legal y médico en diferentes continentes, por ser considerado «un pecado», «un asesinato», «un derecho». No es ilegal en países de la región como Puerto Rico, Guadalupe, Martinica, Estados Unidos y Canadá según comentarios de Miriam Gran, jefa del Departamento de Estadísticas del Ministerio de Salud Pública de Cuba (MINSAP). 
En este sentido, Cuba comenzó el proceso de legalización de este derecho desde finales de la década del 60, ocupándose de garantizar las condiciones más adecuadas para la realización de esta intervención quirúrgica desde los recursos higiénicos y técnicos. Es considerado un derecho humano, que legitima la decisión de la mujer de parir, por todo lo que para ella implica.

Sin embargo, a pesar de la educación sexual que se brinda en el país a través de diversas vías, no se ha logrado del todo el nivel de conciencia necesario para prevenir los embarazos no deseados.

El Estado cuenta con determinadas condiciones que facilitan o no la natalidad, así mismo cada persona con sus posibilidades individualesfamiliares finalmente evalúa su pertinencia. Se trata de una decisión relacional; de pareja, la cual valora y toma o no en cuenta para ello, la red de apoyo que conforma el entramado social en el que viven.

El comportamiento de la fecundidad en Cuba, como se menciona anteriormente, requiere de un análisis holístico, en el que intervienen una compleja gama de factores subjetivos y objetivos que dan al traste con esta situación (ver gráfico 3).

GRÁFICO 3: TASA GLOBAL DE FECUNDIDAD. CUBA, 1992-2007

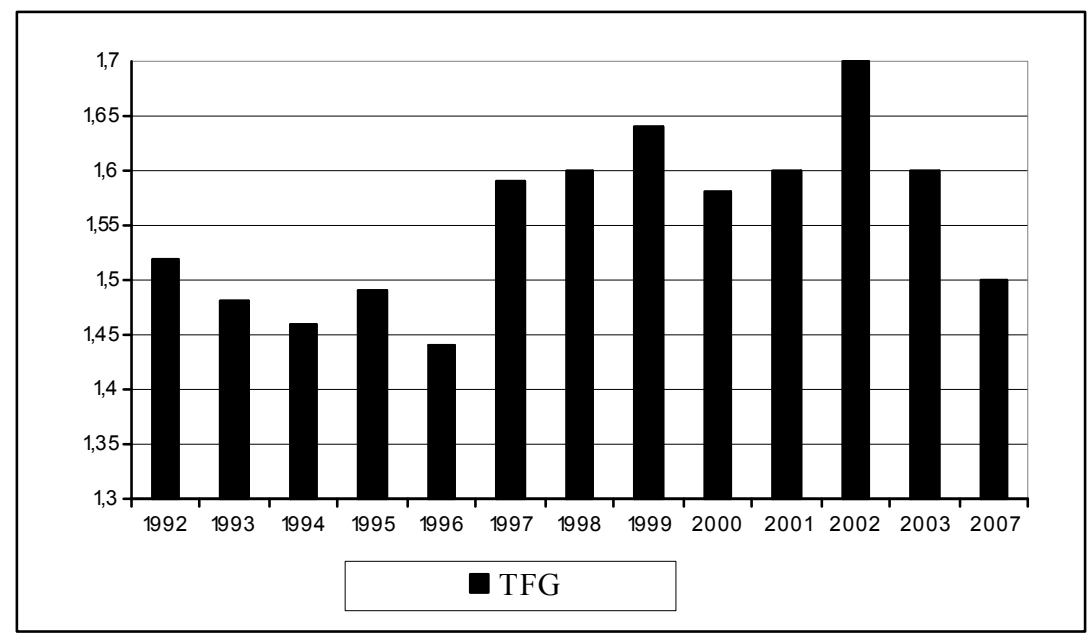

Fuente: MINSAP, 2009.

Los últimos años evidencian una disminución correspondiente a la que se alcanzó hace más de diez años, por lo que resulta impostergable 
repensar las estrategias sociales establecidas. Algunas exploraciones informales adelantan respuestas en esta dirección:

- Un niño cuesta mucho y me gustaría tenerlo cuando pueda brindarle todo lo que necesita.

- No hay suficientes círculos infantiles y no tengo quien me lo cuide.

- La adquisición de una canastilla es muy costosa.

- He priorizado mi vida profesional, cuando llegue a la meta, entonces lo pensaré.

- Es demasiada responsabilidad y no quiero asumirla por ahora.

- No he pensado en tener hijos.

- Solo tendré uno cuando me decida.

- No cuento con espacio ni para poner la cuna.

- Mi pareja no está preparado para tener un hijo.

Estos argumentos indican que el imaginario social se erige sobre argumentos objetivos y subjetivos sin que predominen unos sobre otros. Es de considerar el criterio en el que se combina el comportamiento sexual con la decisión de procrear, pues a veces no se prevé tener descendencia y un descuido determina la maternidad-paternidad. Hay quienes no toman la decisión y esperan por la naturaleza, lo dejan al «azar». Hay quienes tienen descendencia por embullo y otros por presión de los adultos que los rodean.

Lo económico es considerado como un factor objetivo de gran peso ante esta decisión, sin embargo, muchas parejas con buenas condiciones de vida desean posponer su maternidad-paternidad por otros planes asociados a determinados disfrutes. En contraste con ellos, otros en condiciones de vida limitadas tienen más de un hijo.

Por otra parte, se observa un desplazamiento de la maternidad hacia edades más avanzadas, lo cual está asociado a los patrones reproductivos actuales y a las condiciones socioeconómicas por las que ha atravesado el país. En los últimos tiempos se considera que el descenso de la fecundidad ha estado determinado por el mayor impacto de la anticoncepción, el aborto inducido e infertilidad posparto (Gran, 2005).

\section{3. ¿LA DESCENDENCIA, UN CONFLICTO PARA LOS JÓVENES?}

La adolescencia y la juventud constituyen etapas de especial importancia en lo que a sexualidad se refiere, dentro del ciclo vital de cualquier individuo. Para los más jóvenes el proceso es más complejo, 
pues es justamente la época en que se consolidan las transformaciones anatómicas y fisiológicas que ocurren en la pubertad/adolescencia temprana.

Una de las características que podría ponerse de manifiesto es la iniciación temprana de las relaciones sexuales, las que, por lo general, no ocurren con la suficiente preparación que se necesita para asumir sus consecuencias.

Sin embargo, es de mucho valor el arribar a la adolescencia con la adecuada educación sexual, lo cual favorecería un comportamiento responsable. En esta etapa, las relaciones con el otro sexo van siendo cada vez más selectivas, va desapareciendo el isomorfismo.

Se movilizan ante lo socialmente aceptado, les importa «la opinión de los demás iguales», son más críticos hacia la familia, la cual van desplazando hacia un lugar secundario.

La idea de «no vulnerabilidad» es típica de la etapa, les atraen las situaciones de riesgo y suelen coquetear con la curiosidad como móvil de algunas de sus conductas, mucho más en cuestiones relativas a la sexualidad.

Algunos no toman en serio el sexo seguro. Otros asumen actitudes prejuiciados acerca del uso del condón; alegan que les aprieta, que no se siente igual, que no son de buena calidad; otros argumentan que no siempre está disponible para comprarlo, que se les olvida, entre otras expresiones similares, en fin, no lo tienen incorporado como una exigencia de la vida sexual actual.

Esta realidad de no comprensión acerca de la necesidad sistemática del uso del condón, hace vulnerable cualquier proceso de prevención y educación, haciéndolo insuficiente. No obstante, se sigue trabajando en la prevención, no sólo de los embarazos no deseados, sino de las Infecciones de Transmisión Sexual que también están presente dentro de las problemáticas juveniles.

Las dudas, los desencuentros amorosos, los conflictos familiares relacionados con la sexualidad, devienen fuentes de tristeza y depresión, e incluso suelen producirse lesiones auto-inflingidas motivadas, no en pocos casos, por fracasos en la esfera sexual.

La descendencia no constituye una regularidad de estas edades. Cuando ocurre un embarazo, generalmente es por temor a la reacción de la familia, por miedo, desconocimiento, entonces ocultan el embarazo y finalmente se ven precisadas a continuarlo aunque no sea deseado. Hay quienes conciben y consideran natural la descendencia a temprana edad, eso depende de los patrones culturales con los que se 
formó. Las madres adolescentes tienden a ser hijas de mujeres que parieron en su adolescencia.

La educación sexual y reproductiva constituyen una prioridad para los servicios de salud, en tanto las consecuencias de su insuficiencia traen consigo afecciones vinculadas a las regulación de la fecundidad, complicaciones en el parto, práctica del aborto, morbilidad y mortalidad materna, infecciones de transmisión sexual, en especial el VIH/SIDA, infertilidad, entre otras. A su vez, esas afecciones podrían relacionarse con otros comportamientos que se expresan en estas edades tales como conductas adictivas, violentas, problemas nutricionales, etc.

Estas prácticas complejizan el análisis, pues los adolescentes suelen no tener percepción de riesgo como para percatarse de cómo un adicto pierde la noción de peligro, como en estado de embriaguez, pierde el control y no cuida a su pareja y mucho menos cuida de sí mismo en cuanto a la protección que requiere el intercambio sexual.

En etapas como la adolescencia se generan comportamientos de riesgo asociados a la escasa preparación que poseen, sus actitudes y sus prácticas sexuales matizadas por su inexperiencia e interés por explorar vivenciar las nuevas expresiones de su sexualidad.

Justo en estas edades se requieren servicios de salud con un enfoque de género que garanticen la satisfacción de sus necesidades, por lo que avanzar en este sentido favorecería la salud sexual y reproductiva de la sociedad.

Los estudios realizados en el país y concretamente en el CESJ sobre sexualidad en los jóvenes evidencian que muchos comportamientos de riesgo hacen vulnerable las garantías para la salud reproductiva; aún existe desconocimiento relacionado con la planificación familiar y el uso de los métodos anticonceptivos; se inician muy tempranamente las relaciones sexuales sin considerar sus implicaciones, se cambia con frecuencia de pareja, no se le presta atención al hecho de seleccionar pareja, se asumen relaciones sexuales íntimas sin amor y sin protección, etc. (ver gráfico 4). 
GRÁFICO 4: FECUNDIDAD EN LA ADOLESCENCIA. CUBA, 1986-2007

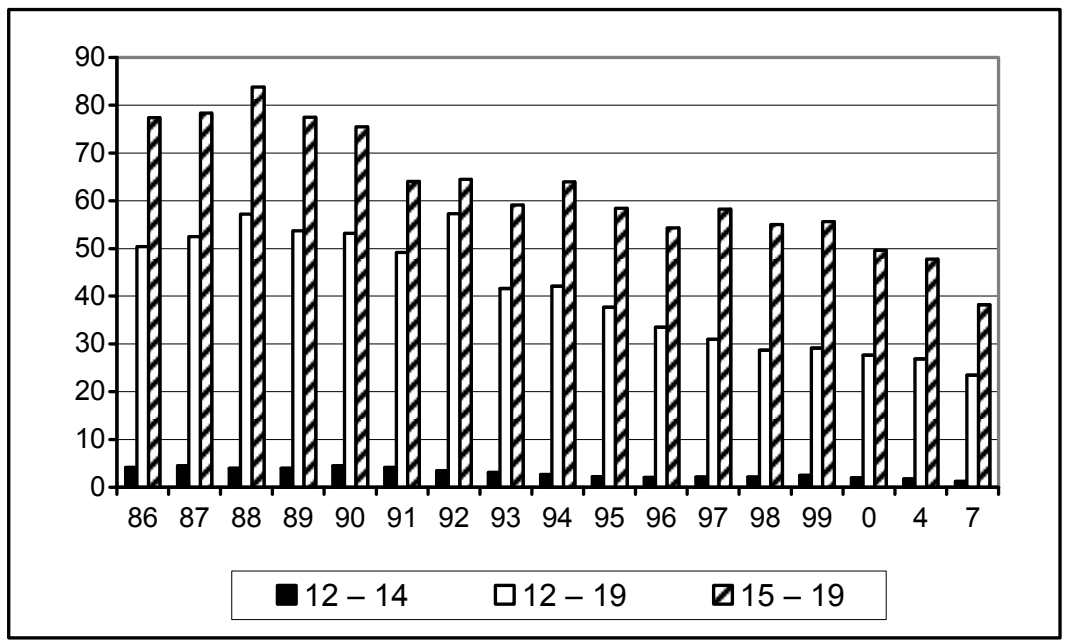

Fuente: MINSAP, 2009.

Especialistas cubanas declaran que muy poco se conoce acerca de la participación del varón en asuntos de fecundidad, en la mayoría de los casos, es la familia de la muchacha la que la acompaña en la toma de la decisión de continuar o no el embarazo, sin que intervenga la pareja sexual (Álvarez, 2009).

A pesar de la intensa labor educativa-preventiva, existe una realidad que requiere un reanálisis de los métodos empleados en la divulgación u otras posibles situaciones que incidan sobre este evento. Se necesita seguir trabajando en esta dirección.

Para los jóvenes propiamente dichos, la esfera sexual goza de mayor estabilidad en cuanto a los cambios anatomofisiológicos que generalmente ocurren en la adolescencia. Aquí aparecen relaciones más estables con el otro sexo, son más selectivos. La elección de la pareja suele ser más espiritual que física. En estas edades se controlan más los impulsos.

Los proyectos de vida devienen regularidad de esta etapa y consideran dentro de ellos la descendencia; tienden a valorar la formación de su nueva familia como una aspiración a mediano o largo plazo.

En este sentido movilizan sus acciones hacia sus prioridades. Ellos jerarquizan estrategias que le brinden salida a sus intereses. Este proceso de ordenamiento personal es realmente complejo, pues va a depender de múltiples situaciones y momentos que requerirán la nego- 
ciación de dos personas, las que no pocas veces entran en conflictos, sobre todo cuando sus proyectos de vida no han sido compartidos, respondiendo sólo a lo individual, o a análisis fragmentados y desestructurados en los que no se han puesto de acuerdo.

En la actualidad, los jóvenes transitan por un largo camino para completar su formación profesional, este motivo es realmente fuerte y en muchos casos decisivo entre los argumentos de posponer la descendencia. Las nuevas tecnologías y exigencias laborales influyen en la toma de decisiones relacionadas con la descendencia.

En Cuba, los jóvenes no gozan tempranamente de total independencia económica por lo antes expuesto, y ésta es otra razón que cuenta al decidir procrear. La familia acompaña y apoya a sus hijos todo el tiempo que sea necesario de manera que ellos avancen en su desarrollo profesional. En no pocas familias se debate sobre el momento adecuado para la llegada del primogénito y también de un segundo hijo.

La experiencia en los estudios acerca del comportamiento sexual de los adolescentes y jóvenes desde el CESJ, ubican algunos elementos asumidos como antecedentes ante la problemática de la fecundidad:

- Inestabilidad en las relaciones de pareja.

- Uso inadecuado de métodos anticonceptivos. Descuidos y olvidos.

- Irregularidad en la distribución de los anticonceptivos y pocos lugares de venta.

- Irresponsabilidad en la selección de la pareja.

- Pobre percepción de riesgo ante las interrupciones de embarazos.

- No tener dentro de su proyecto de vida a corto y mediano plazo la maternidad-paternidad.

- Posponer la maternidad-paternidad para:

- Cuando se concluya la preparación profesional.

- Cuando trabajen.

- Cuando tengan las condiciones financieras creadas.

- Cuando tengan condiciones materiales. Espacio para tenerlo, accesorios para el infante, etc.

Estos argumentos que podrían ser factores que favorecen u obstaculizan la maternidad-paternidad, están inmersos en un entramado social que se refleja en la subjetividad social, expresándose justamente en la posposición de este evento de tanta responsabilidad y trascendencia. Ello significa que no es un hecho aislado el que los jóvenes ofrezcan una $\mathrm{u}$ otra razón para no parir o para posponer este proceso. Existen múltiples factores que lo condicionan. 
La decisión de convertirse en madre y padre necesita ser bien pensada, no sólo se necesita amor y deseos de asumir las responsabilidades que ello engendra. La descendencia es mucho más que un deseo personal, su trascendencia social la coloca entre las decisiones más importantes de la vida, no debería ser un conflicto para los jóvenes.

La garantía de que esto no ocurra implica el empeño de las instituciones, organismos, ministerios que confluyen y participan de alguna manera en que el crecimiento de la población sea una aspiración alcanzable con el esfuerzo y sensibilidad de todas y todos los actores sociales involucrados en tan noble causa.

LA HABANA (CUBA), OCTUBRE 2009

RECIBIDO: OCTUBRE 2009

ACEPTADO: DICIEMBRE 2009

\section{REFERENCIAS BIBLIOGRÁFICAS}

COlectivo DE AUtORES (2009): Salud sexual y reproductiva en adolescentes cubanos. La Habana: Editora Abril.

Figueroa Perea, JuAn Guillermo (S/F): Una aproximación a los derechos sexuales y reproductivos desde la laicidad. México: Colegio de México.

GRAN Álvarez, M. A. (2005): Interrupción voluntaria de embarazo y anticoncepción. Dos métodos de regulación de la fecundidad. Cuba 19952000. La Habana: MINSAP.

MiNSAP (2009): «Planificación familiar en Cuba. Breve reseña programática en el sistema primario de salud». La Habana: MINSAP (inédito).

ONE, CEDEM, CEM (2007): Diagnóstico, escenarios y propuestas de medidas sobre las tendencias demográficas de la población cubana. La Habana: $\mathrm{ONE} / \mathrm{CEDEM} / \mathrm{CEM}$. 